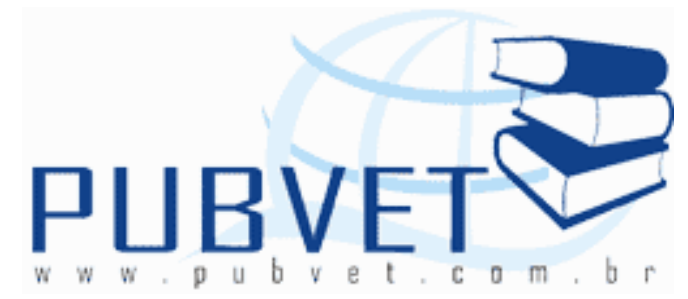

PUBVET, Publicações em Medicina Veterinária e Zootecnia.

\title{
Estudo da vascularização arterial do baço em javalis (Sus scrofa scrofa, linnaeus - 1758)
}

André Luiz Quagliatto Santos, Lucélia Gonçalves Vieira, Líria Queiroz Luz Hirano, Árthur Paulino Sanzo Kaminishi, Juliana dos Santos Mendonça, Thaís Carneiro Santos Rodrigues, Sthéfani Evangelista Siqueira

Laboratório de Ensino e Pesquisa em Animais Silvestres (LAPAS), Universidade Federal de Uberlândia (UFU). Avenida Amazonas 2245, Jardim Umuarama, 38.405-302, Uberlândia-MG.

E-mail: quagliatto@famev.ufu.br

\section{Resumo}

Pesquisas relacionadas a animais silvestres estão crescendo mais a cada dia, obtendo-se informações importantes relacionadas a anatomia, fisiologia e comportamento, essenciais para a sobrevivência dos animais. O baço é um órgão de múltiplas funções sendo responsável pela formação de células sanguíneas, filtração do sangue e resposta imunológica. Apesar de haver muitos estudos sobre a segmentação desse órgão em animais silvestres, não há muitas informações sobre a vascularização deste em Sus scrofa scrofa. Objetivou-se assim descrever a distribuição das artérias em baços de javalis. Para a realização do trabalho utilizou-se 20 órgãos de indivíduos provenientes de criatório. A artéria carótida foi isolada e injetou-se solução de Neoprene Látex. Os animais foram fixados e os baços foram dissecados expondo os 
SANTOS, A.L.Q. et al. Estudo da vascularização arterial do baço em javalis (Sus scrofa scrofa, linnaeus - 1758). PUBVET, Londrina, V. 7, N. 10, Ed. 233, Art. 1540, Maio, 2013.

ramos provenientes da artéria lienal. Em análise dos resultados verificou semelhanças nos estudos realizados com baços de suínos, carnívoros (cães e gatos) e gambás. Houve, no entanto diferença entre as ramificações das artérias lienais entre os gêneros da espécie.

Palavras-chave: anatomia, vascularização, baço, javali.

\title{
Study of arterial vascularization of the spleen in boars \\ (Sus scrofa scrofa, linnaeus - 1758)
}

\begin{abstract}
Related searches to wild animals are growing more each day, yielding important information related to anatomy, physiology and behavior, essential for the survival of the animals. The spleen is an organ with multiple functions being responsible for the formation of blood cells, blood filtration and immune response. Although there are many studies on this organ segmentation in wild animals, there is not much information about this vascularization in Sus scrofa scrofa. The aim of this study was to describe the arteries distribution of the in the spleens of wild boars. To conduct the work we used 20 organs of animals from breeding. The carotid artery was isolated and injected with Neoprene Latex solution. The animals were fixed and spleens were dissected exposing the branches from the splenic artery. Analyzing the results we found similarities spleens characteristics of pigs, carnivores (dogs and cats) and opossums. However there were differences in the branches of the lienais arteries between the gender of the specie.
\end{abstract}

Keywords: anatomy, vascularization, spleen, boar.

\section{INTRODUÇÃO}

Estudos envolvendo animais silvestres estão crescendo cada dia mais. Com este interesse, necessita-se de mais informações sobre a anatomia, 
SANTOS, A.L.Q. et al. Estudo da vascularização arterial do baço em javalis (Sus scrofa scrofa, linnaeus - 1758). PUBVET, Londrina, V. 7, N. 10, Ed. 233, Art. 1540, Maio, 2013.

fisiologia, comportamento e demais aspectos importantes que possam contribuir para a sobrevivência do animal (REGINATO, 2004).

O baço é um órgão de múltiplas funções sendo este responsável pela formação de células sanguíneas, reciclagem do ferro, destruição de eritrócitos velhos e outras partículas, filtração do sangue, armazenamento de sangue, fagocitose e resposta imunológica. Esse órgão compreende ainda a maior parte do tecido linfóide e atua na vida fetal e neonatal como um órgão hematopoiético. No entanto, nos indivíduos adultos o baço não contribui significativamente para tal função (FOZ FILHO, 2001).

A irrigação do baço é feita pela artéria lienal, um ramo calibroso da artéria celíaca (CUSTODIO et al., 1996; DYCE et al., 1990).). Esta ao penetrar no hilo do baço percorre sua superfície visceral emitindo ramos até seu ápice (GHOSHAL, 1981). As afecções esplênicas são de difícil diagnóstico, por seus sinais clínicos inespecifícos e por serem de ocorrência rara. Dentre os relatos clínicos podemos encontrar neoplasias, tromboses, rupturas, esplenomegalia e abcessos (DENNING, BROCKLESBY, 1965).

Muitos autores apresentam estudos sobre a segmentação desse órgão em animais silvestres como Melo (1999), Cavalcante-Filho e colaboradores (1996), Germinaro (1997) e Reginato (2000). O motivo para estudar a vascularização arterial em baço de javalis foi a verificação, nos tratados de Anatomia animal, da existência apenas de descrições genéricas e incompletas sobre a morfologia do baço, não referentes especificamente à distribuição vascular no seu interior.

Visto que o desenvolvimento das técnicas de resseç̧ão parcial dos órgãos depende do conhecimento da segmentação anátomo-cirúrgica, este trabalho tem como objetivo descrever a distribuição das artérias em baços Sus scrofa scrofa. 
SANTOS, A.L.Q. et al. Estudo da vascularização arterial do baço em javalis (Sus scrofa scrofa, linnaeus - 1758). PUBVET, Londrina, V. 7, N. 10, Ed. 233, Art. 1540, Maio, 2013.

\section{MATERIAL E MÉTODOS}

$\mathrm{Na}$ elaboração deste trabalho utilizamos 20 baços de javalis Sus scrofa scrofa, jovens com idade entre 3 a 6 meses, 09 fêmeas e 11 machos, provenientes de um criatório no município de Romaria, Minas Gerais. A artéria carótida comum esquerda foi isolada e colocada uma cânula, posteriormente foi injetada solução corada de Neoprene Látex "450". Os animais foram fixados em solução de formaldeído a $10 \%$ e conservados em tanque de igual solução. Utilizou-se um paquímetro (Starrett/125 B) para efetuar as medições (comprimento, largura e espessura). Posteriormente os baços foram dissecados expondo os ramos provenientes da artéria lienal. Os dados foram registrados em forma de tabelas e desenhos.

\section{RESULTADOS E DISCUSSÃO}

As mensurações realizadas nos baços mostraram um comprimento médio de 106,96 $\mathrm{mm}$ (machos) e 112,69 $\mathrm{mm}$ (fêmeas), \pm 44,63 mm (machos) e $38,47 \mathrm{~mm}$ (fêmeas), a largura média foi de 23,77mm (machos) e 25,80 mm (fêmeas), \pm 9,95 $\mathrm{mm}$ (machos) e \pm 9,17 mm (fêmeas), a espessura média mostrou 8,27 mm (machos) e 7,80 mm (fêmeas) com $\pm 3,97 \mathrm{~mm}$ (machos) e \pm 3,60 mm (fêmeas).

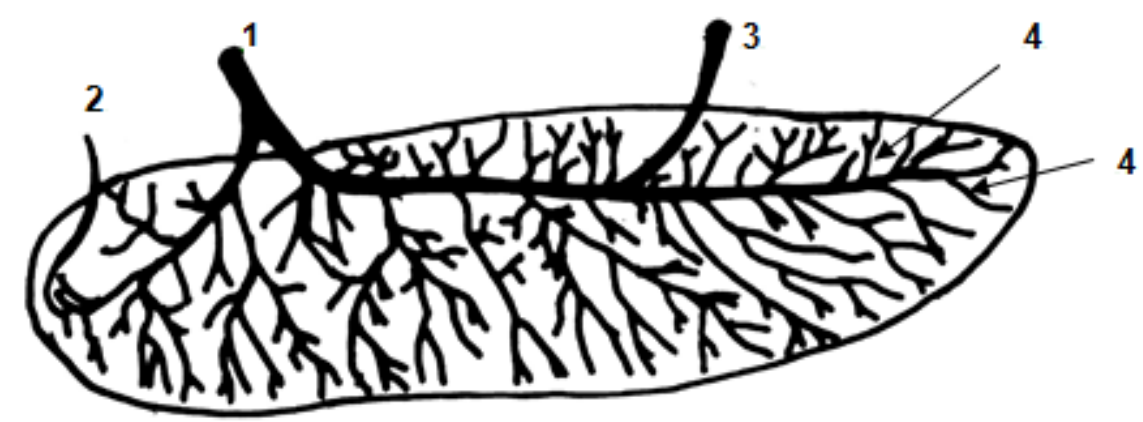

Figura 1: Desenho esquemático do comportamento da artéria lienal (1), no baço de javali, observando o ramo gastrolienal (2), a artéria gástroepiplóica esquerda (3) e os ramos da artéria lienal, tanto craniais quanto caudais, dispondo à semelhança de pente (4). 
SANTOS, A.L.Q. et al. Estudo da vascularização arterial do baço em javalis (Sus scrofa scrofa, linnaeus - 1758). PUBVET, Londrina, V. 7, N. 10, Ed. 233, Art. 1540, Maio, 2013.

A análise dos 20 baços dissecados revelou-nos que em javalis Sus scrofa scrofa, a artéria lienal em todos os casos emitia, antes de penetrar no hilo do baço, um ramo extra-hilar, destinado a irrigação da extremidade dorsal do órgão, abrangendo um território que correspondia, em média, a 19,97 mm do baço. Os baços mostraram de 2 a 4 ramos extra-hilares em Dasypus novemcinctus, L. (tatu-galinha) (SCHIMMING; ABREU, 2001). A artéria lienal, após emitir o ramo extra-hilar, penetrava no hilo esplênico, seguindo o trajeto retilíneo em disposição dorsoventral, cedendo vários ramos para suas margens cranial e caudal. Durante o percurso intraparenquimatoso, a artéria lienal cedia em média 20,7 ramos (machos) e 21 ramos (fêmeas) para a margem cranial e 22,18 ramos (machos) e 26,60 ramos (fêmeas) para a margem caudal do órgão, contrastando com os bovinos da raça nelore que apresentaram, em média 13 ramos para margem cranial e 10 para a margem caudal (CARVALHAL et al., 2001). Em todos os casos, a artéria lienal, emitia ramificações para o omento maior, e estômago, sendo particularmente a artéria gastroepiplóica a mais calibrosa, concordando com os achados em Sus scrofa domesticus (SILVA, MARTINS, 2000).

Considerando a escassez de dados na literatura sobre a vascularização arterial do baço de javalis e até mesmo de suínos, é possível comparar os resultados com aqueles encontrados em outros animais. Em outros estudos foram também utilizados injeção de látex para a visualização da vascularização arterial em suínos (SILVA; MARTINS, 2000). Raios (1982) também utilizou a mesma metodologia em seus estudos com baço de búfalos, bem como Silva (1999) em gambás.

Em contraste, em estudos de segmentos vasculares de búfalos foi realizada uma técnica que envolve a injeção de Butirato de Butilo (GUPTA et al., 1978a). No entanto, observou-se que esse método não preencheu toda a rede vascular, especialmente os vasos mais finos, fato que, segundo Raios (1982) e Silva (1999) podem estar relacionados com a viscosidade do material utilizado. 
SANTOS, A.L.Q. et al. Estudo da vascularização arterial do baço em javalis (Sus scrofa scrofa, linnaeus - 1758). PUBVET, Londrina, V. 7, N. 10, Ed. 233, Art. 1540, Maio, 2013.

Tabela 01: Valores individuais de comprimento, largura e espessura e número de ramos craniais e caudais provenientes da artéria lienal, em baços de Sus scrofa scrofa. Uberlândia, 2005.

\begin{tabular}{|c|c|c|c|c|c|c|}
\hline $\begin{array}{c}\text { № do } \\
\text { animal }\end{array}$ & Sexo & Comp./mm & Larg./mm & Esp./mm & $\begin{array}{l}\text { Ramos } \\
\text { craniais }\end{array}$ & $\begin{array}{l}\text { Ramos } \\
\text { caudais }\end{array}$ \\
\hline 1 & $\mathrm{~F}$ & 85,25 & 15,30 & 5,55 & 21 & 23 \\
\hline 2 & $\mathrm{~F}$ & 151,45 & 32,70 & 12,00 & 17 & 27 \\
\hline 3 & $\mathrm{~F}$ & 72,30 & 14,4 & 3,45 & 22 & 21 \\
\hline 4 & $\mathrm{~F}$ & 134,10 & 28,75 & 6,85 & 28 & 32 \\
\hline 5 & $F$ & 153,30 & 35,1 & 12,05 & 21 & 27 \\
\hline 6 & $\mathrm{~F}$ & 168,70 & 37,70 & 10,80 & 18 & 23 \\
\hline 7 & $F$ & 87,80 & 24,60 & 8,60 & 19 & 22 \\
\hline 8 & $\mathrm{~F}$ & 76,15 & 17,85 & 3,20 & 22 & 22 \\
\hline 9 & $\mathrm{~F}$ & 85,20 & 22,55 & 7,80 & 17 & 19 \\
\hline Média & & 112,69 & 25,80 & 7,80 & 21 & 26,60 \\
\hline $\begin{array}{l}\text { Desvio } \\
\text { padrão }\end{array}$ & & 38,47 & 9,17 & 3,60 & 3,38 & 3,70 \\
\hline 10 & $M$ & 177,45 & 38,55 & 10,75 & 20 & 25 \\
\hline 11 & $M$ & 62,25 & 13,40 & 6,80 & 21 & 20 \\
\hline 12 & $M$ & 104,70 & 22,65 & 4,50 & 19 & 20 \\
\hline 13 & M & 111,25 & 26,85 & 8,50 & 22 & 25 \\
\hline 14 & $M$ & 106,90 & 23,70 & 8,30 & 20 & 22 \\
\hline 15 & $M$ & 105,80 & 24,10 & 6,80 & 20 & 21 \\
\hline 16 & $M$ & 60,35 & 13,10 & 3,85 & 23 & 24 \\
\hline 17 & M & 107,65 & 23,50 & 17,25 & 22 & 29 \\
\hline 18 & $M$ & 153,60 & 36,40 & 10,05 & 16 & 22 \\
\hline 19 & $M$ & 58,50 & 13,10 & 5,65 & 17 & 16 \\
\hline 20 & $M$ & 62,50 & 13,50 & 5,05 & 22 & 22 \\
\hline Média & & 106,93 & 23,77 & 8,27 & 20,27 & 22,18 \\
\hline $\begin{array}{l}\text { Desvio } \\
\text { padrão }\end{array}$ & & 44,63 & 9,95 & 3,97 & 2,19 & 3,45 \\
\hline
\end{tabular}

Em estudos com diferentes espécies de animais com cães (GUPTA et al., 1978a) , cabras (GUPTA et al., 1978b), caprinos (GUPTA et al., 1978c; RAYS et al., 1981), ovelhas (RAYS, 1982) e búfalos, gambás (SILVA, 1999). Nestes estudos foram observados que a divisão do baço é feita com base na distribuição vascular arterial e da segmentação arterial distribuída através do orgão, caracterizando a variabilidade de uma série de extra-ramos hilares da artéria esplênica original dessas espécies. O baço desses animais também apresentaram uma segmentação baseada em ramificações arteriais, sendo que 
SANTOS, A.L.Q. et al. Estudo da vascularização arterial do baço em javalis (Sus scrofa scrofa, linnaeus - 1758). PUBVET, Londrina, V. 7, N. 10, Ed. 233, Art. 1540, Maio, 2013.

na maioria dos casos eles também relataram um baço pedículo. Em contraste com os animais já citados, em porcos, a artéria esplênica segue seus próprios padrões, percorrendo através do hilo e abastecendo as diferente regiões do orgão (SILVA; MARTINS, 2000).

Em análise dos resultados dos estudos encontrados, pesquisas relacionadas ao baço de suínos (SILVA; MARTINS, 2000), em carnívoros (cães e gatos) e os realizados em gambás (SILVA, 1999) apresentaram resultados mais próximos a espécie Sus scrofa scrofa. Zimmerl (1930) e Evans e Christensen (1979) consideraram a artéria como o ramo principal do órgão, fornecendo pequenos ramos para diferentes regiões. Para Reighard e Jennings (1935) e Taylor e Maber (1958) o baço é irrigado por dois ramos, um cranial e um caudal. Crouch (1969) noticiou a presença, além dos ramos citados, a presença de um ramo direito e um esquerdo. Já Zimmerl (1930), Snook (1950), Lewis (1957) e Smalwold e Sis (1973) mencionou inúmeros ramos sem relatar detalhes sobre o arranjo dessas estruturas.

Em estudos sobre búfalos (RAYS, 1982), foram detectadas finas redes de capilares perto do hilo em diferentes segmentos dos ramos primários e secundários. Nos estudos com gatos (HOLZCHUHU, 1977) foram detectadas importantes anastomoses entre os ramos provenientes da artéria esplênica, das artérias gastroepiplóica esquerda e direita, artérias gástricas curtas, ramos colaterais da artéria gástrica esquerda e entre os vasos da artéria esplência em si. No entanto, não foram observadas anastomoses nos vasos analisados do baço de javali.

\section{CONCLUSÕES}

Em análise dos resultados verificou semelhanças nos estudos realizados com baço de suínos, carnívoros (cães e gatos) e gambás. Diante do exposto acima podemos concluir que no baço de javalis Sus scrofa scrofa o hilo esplênico situa-se na superfície visceral, sendo que a artéria lienal antes de penetrar no hilo, emite um ramo extra-hilar. Nos machos, a artéria lienal 
SANTOS, A.L.Q. et al. Estudo da vascularização arterial do baço em javalis (Sus scrofa scrofa, linnaeus - 1758). PUBVET, Londrina, V. 7, N. 10, Ed. 233, Art. 1540, Maio, 2013.

emite em média 20,70 ramos para a margem cranial e 22,18 ramos para a margem caudal enquanto que nas fêmeas emite 21 ramos para a margem cranial e 26,6 para a margem caudal. Estes estão presentes em todos os casos ramos gastrolienais, situados próximos à extremidade dorsal do órgão.

\section{REFERÊNCIAS BIBLIOGRÁFICAS}

CARVALHAL, R.; SOUZA, W.M; MIGLINO, M. A. Artéria e veia lienais de bovinos da raça Nelore. Braz. J. vet. Res. anim. Sci., v. 38, n. 1, p. 09-12, 2001.

CAVALCANTE-FILHO, M. F. et al., Suprimento arterial do baço em queixadas (Tayassu pecari). In: CONGRESSO BRASILEIRO DE ANATOMIA, 17. 1996, Fortaleza, Anais... (Fortaleza: Sociedade Brasileira de Anatomia, 1996. p. 94).

CROUCH, J. E. Text atlas of cat anatomy. Philadelphia, Lea \& Febriger, p.225, 1969.

CUSTÓDIO, A. A et al., Distribuição da artéria esplênica no baço em suínos (Sus scrofa domesticus, Linnaeus-1758). In: Congresso Brasileiro de Anatomia, 17, Fortaleza. Anais... Fortaleza: Sociedade Brasileira de Anatomia, p.110, 1996.

DENNIG, H.K.; BROCKLESBY, D.W. Splenectomy of horses and donkeys. The Veterinary Record, v. 77, n. 2, p. 40-43, 1965.

DYCE, K.M.; SACK, W. O.; WENSING, C. J. G. Tratado de anatomia veterinária. Rio de Janeiro: Guanabara Koogan, p. 349-350, 1990.

EVANS \& CHRISTENSEN, G.C. Miller's anatomy of the dog. 2.ed. Philadelphia, W.B. Saunders, p. 502-503, 1979.

FOZ FILHO, R. P. P. Segmentos anátomo-cirúrgicos do baço do equino (Equis caballus, Linnaeus 1758). 2001. 77f. Tese (Doutorado em Anatomia dos Animais Domésticos) Faculdade Medicina Veterinária e Zootecnia, Universidade de São Paulo. São Paulo, 2001.

GERMINARO, A. et al. A segmentação arterial do baço de capibara (Hidrochoerus hidrochoerus). Brazilian Journal of Veterinary Research and Animal Science São Paulo, v. 34, n. 4, p. 196-202, 1997.

GHOSHAL, M.G. Coração e artérias. In: GETTY, R.; Sisson/Grossman. Anatomia dos animais domésticos. 5 ed. Rio de Janeiro: Guanabara Koogan, v. 1, p.559-560, 1981.

GUPTA, S. C.; GUPTA, C. A. \& GUPTA, S. B. Segmentation in the dog spleen. A study by corrosin cast. Acta anat., 101:380-2, 1978a.

GUPTA, S. C.; GUPTA, C. S.; GUPTA, S. B. Arterial segmentation in the goat (Capra hircus) spleen. A study by corrosion cast. Acta anat., 102:102-4, 1978b.

GUPTA, S. C.; GUPTA, C. S.; ARORA, A. \& GUPTA, S. B. Vascular segments in the buffalo (Bubalus bubalis) spleen. Anat. Anz., 143:393-5, 1978c.

HOLZCHUH, M. P. Irrigação arterial do baço de gato (Felis catus, 1758). Estudo experimental anátomo-fisiológico. Rev. Fac. Med. Vet. Zootec. Univ. São Paulo, 14:205-30, 1977.

LEWIS, D. J. The blood vesseles of the adult mammalian spleen. J. Anat., 91:245-50, 1957. 
MELO, A. P. F. Segmentos anátomo-cirúrgicos arteriais do baço de cervídeos. 1999. 95f. Tese (Doutorado em Anatomia dos Animais Domésticos) - Curso de Pós-Graduação em Anatomia dos Animais Domésticos - Faculdade de Medicina Veterinária e Zootecnia, Universidade de São Paulo, São Paulo, 1999.

RAYS, M. A. A. Estudo da vascularização arterial do baço de búfalo da raça murrah. Ribeirão Preto, 1982. 81 p. Dissertação (Doutorado) - Faculdade de Medicina de Ribeirão Preto, Universidade de São Paulo, Brasil.

RAYS, M.A.A.; DAYOUB, M.C.O.; ORSI, A.M.; DIAS, S.M. Estudo macroscópico da vascularização arterial do baço em caprinos (Capra hireus). In: Jornada Científica, 10, Botucatu, 1981a. Anais. Botucatu, Associação dos Docentes do Campus de Botucatu/UNESP, $1981 a$, p.113.

REGINATO, A. L. et al. Distribuição da artéria lienal no parênquima do baço de queixada (Tayassu pecari, LINK - 1795). Brazilian Journal of Morphological Science, v. 17 (Supl.), p. 144, 2000.

REGINATO, A. L. et al., Segmentação anátomo-cirúrgica em baços de queixada ( Tayassu pecari - Link, 1975). Brazilian Journ al of Veterinary Research and Animal Science, $v$. 41 , p. 281-285, 2004.

REIGHARD, J. \& JENNINGS, H. S. Anatomy of the cat. 3. ed. New York, Hern Holt, p. 303, 1935.

SCHIMMING,B. C. \& ABREU, M. A. F. Systematization of the arteries in the splenic hilus of the armadillo (Dasypus novemcinctus, L.). Rev. chil. anat. v.19 n.2 Temuco ago. 2001.

SILVA, J. R. C. \& MARTINS, M. R. F . B. Comparative study of arterial vascularization in swine spleen (Sus scrofa domesticus). Rev. Chil. Anat., v. 18, n.2, p.223-226, 2000.

SILVA, J. R. C. P. Arterial supply to the spleen of the opossum (Didelphis albiventris). Braz. J. morphol. Sci., 16:79-81, 1999.

SMALWOOD, J. E.; SIS, R. F. Selective arteriography in the cat. Am. J. Vet. Res., 34:955-63, 1973.

SNOOK, T. A comparative study of the vascular arrangements in mammalian spleens. Am. J. Anat., 87:31-69, 1950.

TAYLOR, W. J. \& WEBER, R. J. Functional mammalian anatomy with special reference to the cat. 3.ed. Princepton, D VAN NORTRAND, 1958, p. 305.

ZIMMERL, U. Trattado di anatomia veterinária. Milano: Vallardi, 1930, v.2, p.159. 